\section{Impacto da doença periodontal na qualidade de vida de indivíduos diabéticos dentados}

\author{
Impact of periodontal disease on quality of life for \\ dentate diabetics
}

\author{
${ }^{1}$ Faculdade de Odontologia, \\ Pontifícia Universidade \\ Católica de Minas Gerais, \\ Belo Horizonte, Brasil. \\ Correspondência \\ T. Drumond-Santana \\ Faculdade de Odontologia, \\ Pontifícia Universidade \\ Católica de Minas Gerais. \\ Praça Dr. Augusto Gonçalves \\ 146, sala 907, Itaúna, $M G$ \\ 35680-054, Brasil. \\ triciadrumond@uol.com.br
}

\begin{abstract}
The aim of this study was to evaluate the potential impact of periodontal disease on quality of life in diabetics. A total of 159 dentate diabetic individuals registered at the Municipal Hospital in Itaúna, Minas Gerais, Brazil, were examined and interviewed. The clinical periodontal parameters recorded were: gingival bleeding, probing depth, and clinical attachment level. The OHIP-14 form was used to evaluate the impact of periodontal disease on quality of life. In relation to periodontal status, $15.7 \%$ of individuals were healthy, $35.2 \%$ presented gingivitis, and $49.1 \%$ periodontitis (27.7\% in the mild-to-moderate and $21.4 \%$ in the advanced stages). Association between diagnosis of periodontal disease and impact on quality of life was significant in individuals with periodontitis $(p<0.001)$. Gingival bleeding, probing depth, and clinical attachment level $\geq 4 \mathrm{~mm}$ were associated with intensely negative impact on quality of life ( $p$ $=0.013, p<0.001$, and $p=0.012$ respectively). Diabetics with mild-to-moderate and advanced periodontitis had more negative impact on quality of life than those who were periodontally healthy or with gingivitis.
\end{abstract}

Diabetes Mellitus; Periodontitis; Gengivitis; Oral Health; Quality of Life
Trícia Drumond-Santana 1 Fernando Oliveira Costa 1 Elton Gonçalves Zenóbio 1 Rodrigo Villamarim Soares 1 Taciana Drumond Santana 1

\section{Introdução}

O diabetes mellitus é considerado pela Organização Mundial da Saúde e pela Federação Internacional de Diabetes como uma epidemia global, que atinge aproximadamente 194 milhões de pessoas no mundo ${ }^{1}$. No Brasil, estima-se que $7,6 \%$ da população, na faixa etária de 30 a 69 anos, sejam portadores dessa enfermidade sistêmica ${ }^{2}$.

$\mathrm{O}$ relacionamento entre diabetes mellitus e doença periodontal tem sido extensivamente estudado. Entretanto, é importante ressaltar que as investigações consideraram apenas mensurações do estado de saúde bucal obtidas por meio de observações clínicas diretas, sem levar em conta a autopercepção do indivíduo. Por outro lado, tem sido considerado que a autopercepção em saúde bucal, apesar de subjetiva, é uma medida adicional que contribui para a avaliação dos cuidados em saúde ${ }^{3}$. Esses indicadores subjetivos buscam avaliar o impacto da saúde na qualidade de vida. No campo da saúde bucal, fatores como vida social, alimentação, atividades diárias, bem-estar do indivíduo, entre outros, podem ser afetados por problemas de origem bucal 4 .

Nos últimos anos, foram desenvolvidos vários instrumentos capazes de mensurar a relação entre saúde bucal e qualidade de vida. Isso traduz o crescente reconhecimento de que a saúde bucal pode ocasionar impactos na qualidade de vida. Conseqüentemente, faz-se necessária a inclusão 
desses instrumentos em levantamentos futuros de saúde bucal 5 .

A influência da saúde bucal na qualidade de vida de diabéticos foi investigada utilizando-se o índice de saúde geral SF-36. Observou-se que fatores como insatisfação com os dentes ou a boca e xerostomia foram capazes de influenciar negativamente a qualidade de vida 6 .

Sabendo-se que a doença periodontal tem maior prevalência e gravidade em indivíduos diabéticos quando comparados aos indivíduos não-diabéticos 7 e que o impacto na vida diária da doença periodontal nestes indivíduos não é relatado na literatura, o objetivo deste estudo foi avaliar o impacto dessa doença na qualidade de vida de portadores de diabetes mellitus.

\section{Métodos}

Participaram do estudo epidemiológico transversal 322 diabéticos, de ambos os gêneros, faixa etária de 14-85 anos, matriculados e agendados para controle médico no segundo semestre de 2004, no Departamento de Endocrinologia do Hospital Dr. Ovídio Nogueira Machado, em Itaúna, Minas Gerais, Brasil. Dentre estes, 141 diabéticos eram edêntulos totais e não foram incluídos neste estudo. O Departamento de Endocrinologia do referido hospital possui 1.653 diabéticos cadastrados desde o ano de 1992. Entretanto, a freqüência do atendimento anual desses indivíduos é altamente variável devido a diversos motivos como: óbito, migração, controle inadequado do diabetes mellitus ou realização do controle desta doença em outras localidades. Os 322 diabéticos examinados foram aleatoriamente selecionados.

Os diabéticos foram examinados e entrevistados nas dependências do hospital, em sala arejada contendo pia, utilizando-se cadeira de coleta para exame laboratorial e luz artificial. Todas as entrevistas foram realizadas antes do exame clínico periodontal. Primeiramente, em um estudo piloto, trinta diabéticos foram examinados visando à calibração da examinadora nos critérios do exame periodontal e da utilização do questionário sobre qualidade de vida. Todos os participantes deste estudo piloto foram excluídos da amostra final.

As características do diabetes mellitus (tipo, duração, tratamento e controle da doença) foram obtidas e conferidas do prontuário médico referente à última consulta no momento do exame periodontal.

Para o exame clínico intrabucal foram necessários: espelho bucal, pinça de algodão, sonda periodontal de Williams (Hu-Friedy ${ }^{\circledR}$, MGF Co. Inc., Chicago, Estados Unidos), compressas de gaze e roletes de algodão esterilizados em autoclave.

O índice de sangramento gengival de Ainamo \& Bay ${ }^{8}$ foi utilizado como padrão para avaliação do sangramento gengival à sondagem.

A profundidade de sondagem foi mensurada por meio da sondagem manual circunferencial. Esse parâmetro foi registrado em quatro sítios por dente: vestibular, mesial, distal e lingual ou palatino.

O nível clínico de inserção $\geq 4 \mathrm{~mm}$ foi registrado em quatro sítios por dente: vestibular, mesial, distal e lingual ou palatino.

A doença periodontal foi classificada como: (a) gengivite: indivíduos com a presença de pelo menos um sítio com sangramento à sondagem ${ }^{9}$; (b) periodontite: indivíduos com pelo menos quatro sítios com profundidade de sondagem e nível clínico de inserção $\geq 4 \mathrm{~mm}{ }^{10}$. Sendo que na presença de profundidade de sondagem e nível clínico de inserção $\geq 4 \mathrm{~mm} \mathrm{e} \leq 6 \mathrm{~mm}$, os indivíduos foram classificados como periodontite leve a moderada, e periodontite avançada na presença de sítios com profundidade de sondagem e nível clínico de inserção > 6mm. Após a aplicação do critério para o diagnóstico de periodontite, a determinação da gravidade ocorria pela presença de pelo menos um sítio com a medida de profundidade de sondagem alterada 11 .

Para reduzir os erros inerentes ao processo de sondagem, foram adotados os seguintes critérios de exclusão: dentes com lesão cariosa extensa; procedimentos restauradores iatrogênicos que impedissem a realização do exame adequadamente; alteração da morfologia gengival, com o intuito de minimizar a classificação equivocada de bolsas gengivais em bolsas periodontais e terceiros molares 12 .

Os diabéticos responderam a um formulário antes do exame clínico periodontal, no formato entrevista, cujo objetivo foi avaliar se as alterações da condição bucal, incluindo a doença periodontal, interferiram na qualidade de vida nos últimos 12 meses. Para tanto, foi utilizado o instrumento OHIP-14 (Oral Health Impact Profile) 13, com apenas uma modificação. Como havia o interesse de investigar a repercussão da doença periodontal, acrescentou-se a palavra "gengiva" em 13 perguntas desse índice, exceto na pergunta 3 (Tabela 1).

É importante considerar que o OHIP-14 já foi testado e validado para o uso na cultura e na língua nacional 14 . Além disso, a versão portuguesa do OHIP-14 mostrou boas propriedades psicométricas, semelhantes às exibidas pelo instrumento original. Portanto, não houve a necessidade de validar o instrumento aplicado nesta pesquisa, tendo em mente que este instrumento 


\section{Formulário OHIP-14}

1. Você teve problemas para falar alguma palavra por causa de problemas com seus dentes, sua boca ou gengiva?

2. Você sentiu que o sabor dos alimentos ficou pior por causa de problemas com seus dentes, sua boca ou gengiva?

3. Você sentiu dores em sua boca ou nos seus dentes?

4. Você se sentiu incomodado ao comer algum alimento por causa de problemas com seus dentes, sua boca ou gengiva?

5. Você ficou preocupado por causa de problemas com seus dentes, sua boca ou gengiva?

6. Você sentiu-se estressado por causa de problemas com seus dentes, sua boca ou gengiva?

7. Sua alimentação ficou prejudicada por causa de problemas com seus dentes, sua boca ou gengiva?

8. Você teve que parar suas refeições por causa de problemas com seus dentes, sua boca ou gengiva?

9. Você encontrou dificuldade para relaxar por causa de problemas com seus dentes, sua boca ou gengiva?

10. Você sentiu-se envergonhado por causa de problemas com seus dentes, sua boca ou gengiva?

11. Você ficou irritado com outras pessoas por causa de problemas com seus dentes, sua boca ou gengiva?

12. Você teve dificuldade em realizar suas atividades diárias por causa de problemas com seus dentes, sua boca ou gengiva?

13. Você sentiu que a vida, em geral, ficou pior por causa de problemas com seus dentes, sua boca ou gengiva?

14. Você ficou totalmente incapaz de fazer suas atividades diárias por causa de problemas com seus dentes, sua boca ou gengiva?

já é validado para a população em estudo. Entretanto, para aumentar a confiabilidade dos resultados, nos trinta indivíduos do estudo piloto, os questionários foram reaplicados no intervalo de sete dias e, a confiabilidade teste-reteste foi analisada pelo coeficiente de correlação de Pearson $(0,87 ; \mathrm{p}<0,01)$ e pelo teste alfa de Cronbach's $(0,93)$, sendo que estes resultados mostraram estabilidade e consistência interna, demonstrando a examinadora estar adequada para a aplicação dos mesmos.

A concordância intra-examinador foi realizada utilizando-se o teste de Wilcoxon. Os resultados mostraram que para os parâmetros clínicos profundidade de sondagem e nível clínico de inserção não houve diferenças significativas entre as duas avaliações, podendo concluir que a examinadora apresentou boa capacidade em reproduzir medidas (profundidade de sondagem, $\mathrm{p}=0$,893; nível clínico de inserção, $\mathrm{p}=0$,967).

Para calcular o impacto da doença periodontal na qualidade de vida, pontuações originais do OHIP-14 foram conferidas para cada pergunta de acordo com a resposta apresentada, seguindo o método ponderado padronizado baseado em Allen \& Locker 14, Robinson et al. 15 e Oliveira \& Nadanovsky 16: nunca - 0; raramente - 1 ; às vezes - 2; freqüentemente - 3; sempre - 4; não sabe - exclusão (de todo o formulário).

Além disso, esse valor foi multiplicado pelo peso de cada pergunta como se segue: Pergunta 1: peso $=0,51 ;$ Pergunta 2: peso $=0,49 ;$ Pergunta 3: peso $=0,34 ;$ Pergunta 4 : peso $=0,66$; Pergunta 5: peso $=0,45$; Pergunta 6: peso $=0,55$;
Pergunta 7: peso $=0,52$; Pergunta 8: peso $=0,48$; Pergunta 9: peso $=0,60$; Pergunta 10: peso $=0,40$; Pergunta 11: peso $=0,62$; Pergunta 12: peso $=0,38$; Pergunta 13: peso $=0,59$; Pergunta 14: peso $=0,41$.

Desse modo, ao somarmos a pontuação final de todas as perguntas, obtivemos valores variando entre 0 e 28 pontos. Quanto maior a pontuação apresentada, maior a percepção do impacto pelo diabético ${ }^{13}$.

A análise dos resultados incluiu medidas descritivas (freqüência de distribuição e média) e análise comparativa. Como as medidas de qualidade de vida não foram normalmente distribuídas, testes estatísticos não-paramétricos foram utilizados na análise comparativa (teste KruskalWallis para três ou mais amostras e o teste MannWhitney para duas amostras).

Este estudo esteve de acordo com as normas e diretrizes da Resolução no. 196/96 do Conselho Nacional de Saúde, resolução esta que regulamenta a pesquisa com envolvimento de seres humanos, e foi avaliado e aprovado pelo Comitê de Ética e Pesquisa da Faculdade de Odontologia, Pontifícia Universidade Católica de Minas Gerais. O Departamento de Endocrinologia do Hospital Dr. Ovídio Nogueira Machado autorizou a realização deste estudo em suas dependências.

\section{Resultados}

O formulário OHIP-14 modificado, utilizado neste estudo, foi respondido por 167 dos 181 diabéticos dentados. Ausência de consentimento (sendo 
a principal alegação a falta de tempo para resposta) ocorreu em 14 indivíduos $(7,7 \%)$ e 8 formulários foram excluídos (pelo critério "não sei”). Isso conduz a uma amostra final de 159 diabéticos; 7 de 14-20 anos (4,4\%), 34 de 21-40 (21,4\%), 91 de 41-60 (57,2\%) e 27 acima de 60 anos (17\%). Cento e quatro indivíduos eram do gênero feminino $(65,4 \%)$ e 55 do masculino (34,6\%). Além disso, houve um predomínio de diabéticos do tipo 2 (92,9\%), com até dez anos de diagnóstico do diabetes mellitus $(64,3 \%)$, que eram tratados com insulina $(43,2 \%)$ e apresentaram taxa de glicemia média nos últimos seis meses de 141 a $200 \mathrm{mg} / \mathrm{dl}$ (36,7\%). Complicações do diabetes mellitus, como alterações renal, neurológica ou oftálmica, foram observadas em 40,7\% dos participantes.

Considerando-se o diagnóstico da doença periodontal, 25 indivíduos foram classificados como periodontalmente saudáveis (15,7\%), 56 apresentaram gengivite $(35,2 \%), 44$ periodontite leve a moderada $(27,7 \%)$ e 34 periodontite avançada $(21,4 \%)$.

No geral, observou-se uma variação de 0 a 21,4 pontos para o OHIP-14 modificado, com a seguinte distribuição: $54 \%$ obtiveram de 0 a 3 pontos; $16,8 \%$ de 3,01 a $6 ; 17,4 \%$ de 6,01 a 10 e; $11,8 \%$ de 10,01 a 21,4 pontos. O total de pontos 0 , referente à presença de nenhum impacto negativo, foi verificado em $23 \%$ da amostra.

$\mathrm{Na}$ Tabela 2, a amostra foi dividida conforme o diagnóstico periodontal para expressar os valores do OHIP-14 modificado. As maiores médias do formulário aplicado foram encontradas nos grupos com periodontite, demonstrando que estes indivíduos apresentaram maiores impactos negativos que os demais grupos. Entretanto, não foi observada diferença estatisticamente significativa entre os grupos com periodontite leve a moderada e periodontite avançada, isto é, não houve relação entre gravidade da periodontite e qualidade de vida.
Os grupos saudável e gengivite apresentaram médias similares, sendo estas estatisticamente inferiores aos grupos com periodontite $(\mathrm{p}<$ 0,001). Entre os diabéticos dos grupos saudável e gengivite, aproximadamente $40 \%$ relataram ausência de impacto negativo na qualidade de vida, enquanto que no grupo com periodontite leve a moderada este valor foi de $4,5 \%$, e no grupo com periodontite avançada foi de $8,8 \%$.

Em todos os grupos, as perguntas que obtiveram as maiores médias foram as referentes ao incômodo na mastigação, desconforto pessoal e inibição. Já as referentes à dificuldade para fazer obrigações e total incapacidade de exercer atividades obtiveram as menores médias. Os resultados encontrados para cada uma das 14 perguntas, para a amostra total, estão descritos na Tabela 3.

A descrição dos percentuais de diabéticos que sentiram, nos últimos 12 meses, cada uma das repercussões questionadas, considerando-se o diagnóstico da doença periodontal, está detalhada na Tabela 4.

O teste Mann-Whitney revelou que a presença do sangramento gengival à sondagem obteve associação positiva com a qualidade de vida $(\mathrm{p}=$ 0,013 ), sendo que os diabéticos com sangramento gengival exibiram impactos estatisticamente superiores àqueles que não apresentaram sangramento gengival à sondagem. O mesmo foi encontrado quando esse teste foi aplicado para os parâmetros profundidade de sondagem e nível clínico de inserção. Os grupos com profundidade de sondagem e nível clínico de inserção $\geq 4 \mathrm{~mm}$ apresentaram maiores repercussões negativas sobre a qualidade de vida, $\mathrm{p}<0,001 \mathrm{e} \mathrm{p}=0,012$, respectivamente. Para a verificação dessas associações, foi considerado a soma total do OHIP-14 modificado, e não a associação dos parâmetros clínicos periodontais com cada uma das 14 perguntas do formulário.

Caracterização da amostra quanto às medidas obtidas pelo Oral Health Impact Profile (OHIP-14) modificado, considerando-se o processo saúde-doença periodontal.

\begin{tabular}{|c|c|c|c|c|c|c|}
\hline \multirow[t]{2}{*}{ Diagnóstico } & \multicolumn{5}{|c|}{ Medidas descritivas } & \multirow[t]{2}{*}{$p$} \\
\hline & Mínimo & Máximo & Mediana & Média & Desvio-padrão & \\
\hline Saudável $\left(\mathrm{G}_{\mathrm{S}}\right)$ & 0,0 & 18,8 & 0,5 & 3,3 & 4,8 & \\
\hline Gengivite $\left(\mathrm{G}_{\mathrm{G}}\right)$ & 0,0 & 12,8 & 0,6 & 2,0 & 3,1 & $<0,001$ \\
\hline Periodontite leve a moderada $\left(\mathrm{G}_{\mathrm{PLM}}\right)$ & 0,0 & 15,1 & 4,6 & 5,4 & 4,0 & \\
\hline Periodontite avançada $\left(\mathrm{G}_{\mathrm{PA}}\right)$ & 0,0 & 21,4 & 5,6 & 6,4 & 5,5 & \\
\hline
\end{tabular}

Nota: a probabilidade de significância refere-se ao teste Kruskal-Wallis. Conclusão: $\left(G_{S}=G_{G}\right)<\left(G_{P L M}=G_{P A}\right)$. 
Distribuição das pontuações obtidas por questão (ordem decrescente das médias).

\begin{tabular}{|c|c|c|c|c|c|c|}
\hline \multirow[t]{2}{*}{ Impactos investigados } & \multicolumn{5}{|c|}{ Freqüência (\%) } & \multirow[t]{2}{*}{ Média } \\
\hline & 0 & 1 & 2 & 3 & 4 & \\
\hline Incômodo na investigação & 57,2 & 9,4 & 12,0 & 15,1 & 6,3 & 0,68 \\
\hline Desconforto pessoal & 51,6 & 7,5 & 18,2 & 10,7 & 12,0 & 0,56 \\
\hline Inibição & 51,6 & 13,2 & 9,5 & 11,9 & 13,8 & 0,49 \\
\hline Estresse & 74,2 & 3,8 & 10,1 & 2,5 & 9,4 & 0,38 \\
\hline Bem-estar & 78,6 & 3,8 & 8,2 & 6,9 & 2,5 & 0,30 \\
\hline Dor & 56,0 & 14,5 & 19,5 & 6,9 & 3,1 & 0,29 \\
\hline Incômodo na alimentação & 78,6 & 3,1 & 9,4 & 5,1 & 3,8 & 0,27 \\
\hline Irritação com outras pessoas & 83,6 & 3,8 & 4,4 & 1,9 & 6,3 & 0,26 \\
\hline Dificuldade para relaxar & 83,0 & 2,5 & 9,5 & 2,5 & 2,5 & 0,23 \\
\hline Diminuição do paladar & 78,0 & 5,7 & 13,2 & 0,0 & 3,1 & 0,22 \\
\hline Interrupção das refeições & 85,5 & 2,5 & 8,8 & 0,7 & 2,5 & 0,15 \\
\hline Pronúncia de palavras & 89,9 & 2,5 & 3,8 & 3,1 & 0,7 & 0,11 \\
\hline Dificuldade para fazer obrigações & 92,5 & 3,7 & 3,1 & 0,0 & 0,7 & 0,05 \\
\hline Total incapacidade de exercer atividades & 96,9 & 1,2 & 0,0 & 1,2 & 0,7 & 0,03 \\
\hline
\end{tabular}

$0=$ nunca; 1 = raramente; 2 = às vezes; 3 = freqüente; 4 = sempre; exclusão = não sei.

Tabela 4

Percentual de indivíduos afetados pelos impactos investigados nos últimos 12 meses (ordem decrescente).

\begin{tabular}{|c|c|c|c|c|c|}
\hline \multirow[t]{2}{*}{ Impacto investigado } & \multicolumn{4}{|c|}{ Indivíduos afetados (\%) } & \multirow[t]{2}{*}{ Total } \\
\hline & $\mathrm{G}_{\mathrm{S}}$ & $\mathrm{G}_{\mathrm{G}}$ & $\mathrm{G}_{\mathrm{PLM}}$ & $\mathrm{G}_{\mathrm{PA}}$ & \\
\hline Inibição & 36,0 & 32,1 & 65,9 & 61,8 & 48,4 \\
\hline Desconforto pessoal & 36,0 & 28,6 & 70,5 & 61,8 & 48,4 \\
\hline Dor & 36,0 & 30,4 & 54,5 & 58,8 & 44,0 \\
\hline Incômodo na mastigação & 32,0 & 25,0 & 59,1 & 58,8 & 42,8 \\
\hline Estresse & 12,0 & 8,9 & 40,9 & 44,1 & 25,8 \\
\hline Diminuição do paladar & 8,0 & 6,1 & 25,0 & 38,2 & 22,0 \\
\hline Incômodo na alimentação & 16,0 & 10,7 & 31,8 & 29,4 & 21,4 \\
\hline Bem-estar & 16,0 & 8,9 & 29,5 & 35,3 & 21,4 \\
\hline Dificuldade para relaxar & 12,0 & 8,9 & 20,5 & 29,4 & 17,0 \\
\hline Irritação com outras pessoas & 16,0 & 5,4 & 21,5 & 29,4 & 16,4 \\
\hline Interrupção das refeições & 16,0 & 10,7 & 31,8 & 29,4 & 14,5 \\
\hline Pronúncia de palavras & 8,0 & 1,6 & 18,2 & 14,7 & 10,1 \\
\hline Dificuldade para fazer obrigações & 8,0 & 7,1 & 6,8 & 8,8 & 7,5 \\
\hline Total incapacidade de exercer atividades & 4,0 & 1,8 & 2,3 & 5,9 & 3,1 \\
\hline
\end{tabular}

$\mathrm{G}_{\mathrm{S}}=$ saudável; $\mathrm{G}_{\mathrm{G}}=$ gengivite; $\mathrm{G}_{\mathrm{PLM}}=$ periodontite leve a moderada; $\mathrm{G}_{\mathrm{PA}}$ = periodontite avançada. O percentual refere-se aos indivíduos que responderam de forma positiva para a ocorrência do impacto nos últimos 12 meses, independente da freqüência com que foi sentido. Foram considerados afetados todos os indivíduos que responderam raramente, às vezes, freqüentemente ou sempre. 


\section{Discussão}

O instrumento OHIP-14 é considerado atualmente um bom indicador para captar percepções e sentimentos dos indivíduos sobre sua própria saúde bucal e suas expectativas em relação ao tratamento e serviços odontológicos, tornandose uma metodologia de escolha em avaliações com esta finalidade 17 .

Destaca-se que os estudos existentes na literatura sobre qualidade de vida não incluíram portadores de diabetes mellitus. Além disso, poucos estudos, como o de Leão et al. 4, avaliaram a influência da doença periodontal na qualidade de vida, o que torna mais complexa a análise e comparação dos dados.

Os resultados do presente estudo mostraram que aproximadamente $75 \%$ dos diabéticos apresentaram impacto negativo na qualidade de vida em pelo menos uma pergunta. Esses achados se assemelham aos encontrados por Adulyanon et al. 18 , que obtiveram valores de $73,6 \%$ em uma amostra de 501 tailandeses de 35-44 anos, mas são superiores aos relatados por Leão et al. 4, Masalu \& Astrom 19 e Locker et al. 20 , nos quais aproximadamente 50 a $60 \%$ dos entrevistados tiveram impacto em pelo menos uma pergunta. Leão et al. 4 e Locker et al. 20 avaliaram a percepção dos impactos nos últimos três meses e Masalu \& Astrom 19 investigaram os últimos seis meses. Este estudo avaliou a percepção dos impactos nos últimos 12 meses, o que justifica o maior valor encontrado.

Comparando-se este estudo com outros 13,21,22 que também utilizaram o formulário OHIP-14, pode-se observar que, os últimos apresentaram resultados inferiores nos impactos investigados. Nos estudos citados, os participantes não eram portadores de diabetes mellitus. Estão presentes na literatura estudos que compararam a qualidade de vida de diabéticos com controles não-diabéticos, e estes apresentaram impactos negativos na qualidade de vida intensificados no grupo diabético 6,23.

Em relação à escolha do método ponderado padronizado em detrimento ao método aditivo, já foi estabelecido que estes métodos apresentam alta correlação $\left(r_{s} \geq 0,96\right)$, tendo desempenho semelhante apesar da influência de fatores como idade e diferenças transculturais 14,15,16.

Uma vez que as médias do formulário aplicado dos grupos saudável e gengivite foram similares e estatisticamente inferiores aos grupos com periodontite $(\mathrm{p}<0,001)$, torna-se importante ressaltar que, esta semelhança pode ter ocorrido devido ao fato de que os indivíduos, muitas vezes, não percebem a presença do quadro de gengivite.
Considerando-se o diagnóstico da doença periodontal, os grupos com periodontite, tanto na forma leve a moderada quanto na forma avançada, relataram impactos negativos intensificados na qualidade de vida, entretanto, não foi observada diferença estatisticamente significativa entre estes grupos. Loureiro 24 , empregando uma adaptação do OHIP-14 em crianças e jovens portadores da síndrome de Down, constatou que os impactos negativos acentuaram-se proporcionalmente com a gravidade da doença periodontal, enquanto no atual estudo, não foi observada diferença estatisticamente representativa entre indivíduos saudáveis e com gengivite, assim como entre as formas leve a moderada e avançada de periodontite.

Atualmente, têm sido objeto de muita controvérsia os critérios de definição de caso e gravidade em periodontia. A maioria dos estudos epidemiológicos conduzidos até o final da década de 90 utilizou critérios com baixo ponto de corte nos parâmetros clínicos de profundidade de sondagem e nível clínico de inserção para definição de periodontite e, conseqüentemente, seus achados têm sido apontados como superestimados 7,12. O presente estudo procurou utilizar um critério mais rigoroso no diagnóstico 10,11, assim sendo, pode também ser hipotetizado que esta escolha pode ter influenciado a ausência de associação entre impactos negativos e a gravidade da periodontite.

O questionamento sobre incômodo na mastigação obteve a maior média entre as 14 perguntas do formulário, confirmando o resultado encontrado por Slade 13. Deve ser ressaltado que, esse questionamento apresenta o maior peso dentro do formulário OHIP-14, o que pode ter contribuído para a obtenção desses resultados.

Além de estarem entre as três perguntas com as maiores médias, as perguntas referentes à inibição e desconforto pessoal obtiveram a maior porcentagem de diabéticos afetados. Em estudo realizado na população brasileira, faixa etária $\geq 15$ anos, Dini et al. 25 encontraram resultados semelhantes, apesar dos 323 participantes não apresentarem diabetes mellitus.

Já a respeito do questionamento sobre dor, os resultados de estudos prévios, que também avaliaram a percepção dos impactos nos últimos 12 meses, como os realizados por Slade 13 e Locker \& Allen 21 , detectaram que a dor foi observada entre os quesitos mais afetados por alterações da saúde bucal, o que pode ser admitido para os diabéticos incluídos neste estudo.

Dentro das dimensões desconforto psicológico e incapacidade psicológica, as perguntas referentes a estresse e dificuldade para relaxar 
obtiveram o dobro de impactos negativos, comparando-se com outros estudos 13,21.

Os impactos referentes à dificuldade para fazer obrigações e total incapacidade nas atividades obtiveram as menores porcentagens de diabéticos afetados na amostra total, apesar de que considerando-se o diagnóstico da doença periodontal, somente estes questionamentos não foram mais prevalentes nos indivíduos com periodontite. Esses achados são confirmados pela maioria dos trabalhos que investigaram esses impactos 13,18,21,22.

A presença de sangramento gengival à sondagem, profundidade de sondagem e nível clínico de inserção $\geq 4 \mathrm{~mm}$ associaram-se com impactos negativos intensificados na qualidade de vida (respectivamente, $\mathrm{p}=0,013, \mathrm{p}<0,001 \mathrm{e} \mathrm{p}=0,012$ ). Leão et al. 4 já haviam observado que a presença de alterações periodontais contribui para a intensificação de impactos negativos na vida diária e que a participação dos indivíduos ajuda o profissional a comparar a visão clínica com as necessidades da população, especialmente em relação à qualidade de vida.

Deve ser destacado que no delineamento deste estudo algumas limitações devem ser consideradas, como a presença de outras alterações bucais e comorbidades relacionadas ao diabetes mellitus, que poderiam influenciar nos impactos observados. Entretanto, a análise desses fatores não foi objetivo do presente estudo.

Estudos 4,13,25 prévios sobre repercussão da condição bucal na qualidade de vida demonstraram que alterações da saúde bucal interferem negativamente na qualidade de vida das pessoas afetadas. Os resultados do presente estudo confirmam essas observações e, indicam que é necessário o desenvolvimento de programas específicos com estratégias que minimizem os efeitos negativos da doença periodontal na qualidade de vida de indivíduos portadores de diabetes mellitus.

\section{Resumo}

O objetivo deste estudo foi avaliar a possibilidade da doença periodontal comprometer a qualidade de vida de indivíduos portadores de diabetes mellitus. Foram examinados e entrevistados 159 diabéticos dentados, cadastrados no Hospital Municipal de Itaúna, Minas Gerais, Brasil. Os parâmetros clínicos periodontais registrados foram: sangramento gengival à sondagem, profundidade de sondagem e nível clínico de inserção. A influência da doença periodontal na qualidade de vida foi avaliada utilizando-se o formulário OHIP-14. Em relação à condição periodontal, 15,7\% apresentaram periodonto saudável, 35,2\% gengivite e 49,1\% periodontite, sendo $27,7 \%$ na forma leve a moderada e 21,4\% na avançada. Associação entre diagnóstico da doença periodontal e qualidade de vida foi significati$v a$ nos indivíduos com periodontite $(p<0,001)$. A presença de sangramento gengival à sondagem, profundidade de sondagem e nível clínico de inserção $\geq 4 \mathrm{~mm}$ associaram-se com impactos negativos intensificados na qualidade de vida (respectivamente, $p=0,013$, $p<0,001$ e $p=0,012$ ). Diabéticos com periodontites leve a moderada e avançada apresentaram maiores impactos negativos na qualidade de vida que diabéticos periodontalmente saudáveis ou com gengivite.

Diabetes Mellitus; Periodontite; Gengivite; Saúde Bucal; Qualidade de Vida

\section{Colaboradores}

T. Drumond-Santana participou da coleta de dados, realizou pesquisa bibliográfica, análise dos dados e redigiu o artigo. F. O. Costa planejou e orientou a pesquisa, contribuiu na análise dos dados e na redação do artigo. E. G. Zenóbio, R. V. Soares e T. D. Santana participaram da análise dos dados e revisão do artigo. 


\section{Referências}

1. International Diabetes Federation. What is diabetes? http://www.idf.org/home/index.cfm?node=2 (acessado em 04/Jun/2004).

2. Sociedade Brasileira de Diabetes. Estatísticas do diabetes no Brasil e no mundo. http://www.diabetes. org.br/Diabetes/diabet_set.htm (acessado em 04/ Jun/2004).

3. Sheiham A. A determinação de necessidades de tratamento odontológico: uma abordagem social. In: Pinto VG, organizador. Saúde bucal coletiva. $4 \underline{a}$ Ed. São Paulo: Editora Santos; 2000. p. 223-50.

4. Leão ATT, Cidade MC, Varela JR. Impactos da saúde periodontal na vida diária. Rev Bras Odontol 1998; 55:238-41.

5. Slade GD, Strauss, RP, Atchison KA, Kressin NR, Locker D, Reisine ST. Assessing oral health outcomes-measuring health status and quality of life. Community Dent Health 1998; 15:3-7.

6. Sandberg GE, Wikblad KF. Oral health and healthrelated quality of life in type 2 diabetic patients and non-diabetic controls. Acta Odontol Scand 2003; 61:141-8.

7. Albandar JM. Global risk factors and risk indicators for periodontal diseases. Periodontol 2000 2002; 29:177-206.

8. Ainamo J, Bay I. Problems and proposals for recording gingivititis and plaque. Int Dent J 1975; 25:229-35.

9. Lang NP, Adler R, Joss A, Nyman S. Absence of bleeding on probing: an indicator of periodontal stability. J Clin Periodontol 1990; 17:714-21.

10. Beck JD. Methods of assessing risk for periodontitis and developing multifactorial models. J Periodontol 1994; 65:468-78.

11. American Academy of Periodontology. Parameters of care. J Periodontol 2000; 71:847-83.

12. Armitage GC. Periodontal diseases: diagnosis. Ann Periodontol 1996; 1:37-215.

13. Slade GD. Derivation and validation of a shortform oral health impact profile. Community Dent Oral Epidemiol 1997; 25:284-90

14. Allen PF, Locker D. Do item weights matter? An assessment using the Oral Health Impact Profile. Community Dent Health 1997; 14:133-8.
15. Robinson PG, Gibson B, Klan FA, Birnbaum W. Validity of two oral health-related quality of life measures. Community Dent Oral Epidemiol 2003; 31:90-9.

16. Oliveira BH, Nadanovsky P. Psychometric properties of the Brazilian version of the Oral Health Impact Profile-short form. Community Dent Oral Epidemiol 2005; 33:307-14

17. Miotto MHMB, Barcellos LA. Uma revisão sobre o indicador de saúde bucal "Oral Health Impact Profile” OHIP. UFES Rev Odontol 2001; 3:32-8.

18. Adulyanon S, Vourapukjaru J, Sheiham A. Oral impacts affecting daily performance in a low dental disease Thai population. Community Dent Oral Epidemiol 1996; 24:385-9.

19. Masalu JR, Astrom AN. Social and behavioral correlates of oral quality of life studied among university students in Tanzania. Acta Odontol Scand 2002; 60:353-9.

20. Locker D, Jokovic A, Stephens M, Kenny D, Tompson B, Guyatt G. Family impact of child oral and oro-facial conditions. Community Dent Oral Epidemiol 2002; 30:438-48.

21. Locker D, Allen PF. Developing short-form measures of oral health-related quality of life. J Public Health Dent 2002; 62:13-20.

22. Wong MCM, Lo ECM, McMillan AS. Validation of a Chinese version of the Oral Health Impact Profile (OHIP). Community Dent Oral Epidemiol 2002; 30:423-30.

23. Ahroni JH, Boyko EJ, Davignon DR, Pecoraro RE. The health and functional status of veterans with diabetes. Diabetes Care 1994; 17:318-21.

24. Loureiro ACM. Parâmetros clínicos periodontais e seus impactos na qualidade de vida de portadores da síndrome de Down [Dissertação de Mestrado]. Belo Horizonte: Faculdade de Odontologia, Universidade Federal de Minas Gerais; 2003.

25. Dini EL, McGrath C, Bedi R. An evaluation of the oral health quality of life (OHQoL) instrument in a Brazilian population. Community Dent Health 2003; 20:40-4.

Recebido em 16/Set/2005

Versão final reapresentada em 22/Mai/2006

Aprovado em 01/Ago/2006 